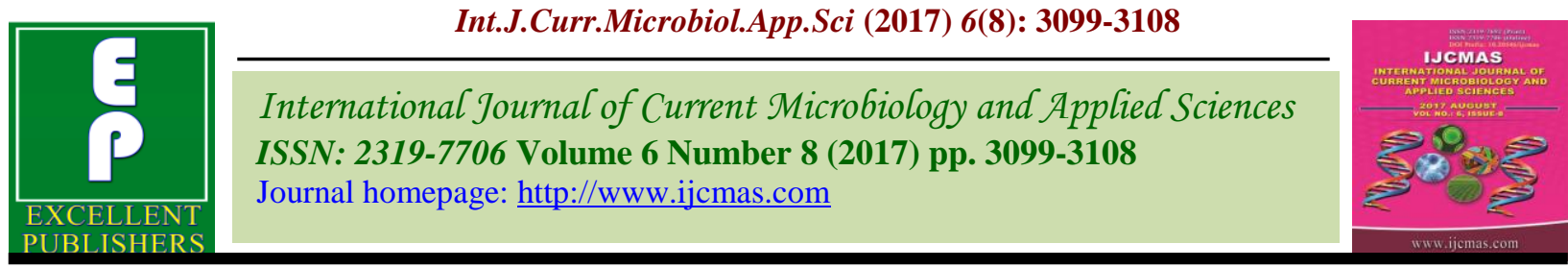

Original Research Article

https://doi.org/10.20546/ijcmas.2017.608.371

\title{
Effect of Various Nitrogen Sources on Microalgal Growth and Lipid Content in Chlorella pyrenoidosa NCIM 2738 and ANK-1
}

\author{
Sweta M. Soni ${ }^{*}$, Sunny S. Sankneniwar, M.A. Rasheed, P.L.S. Rao and S.Z. Hasan \\ Petroleum Research Wing, Gujarat Energy Research and Management Institute (GERMI), \\ Gandhinagar, Gujarat, India \\ *Corresponding author
}

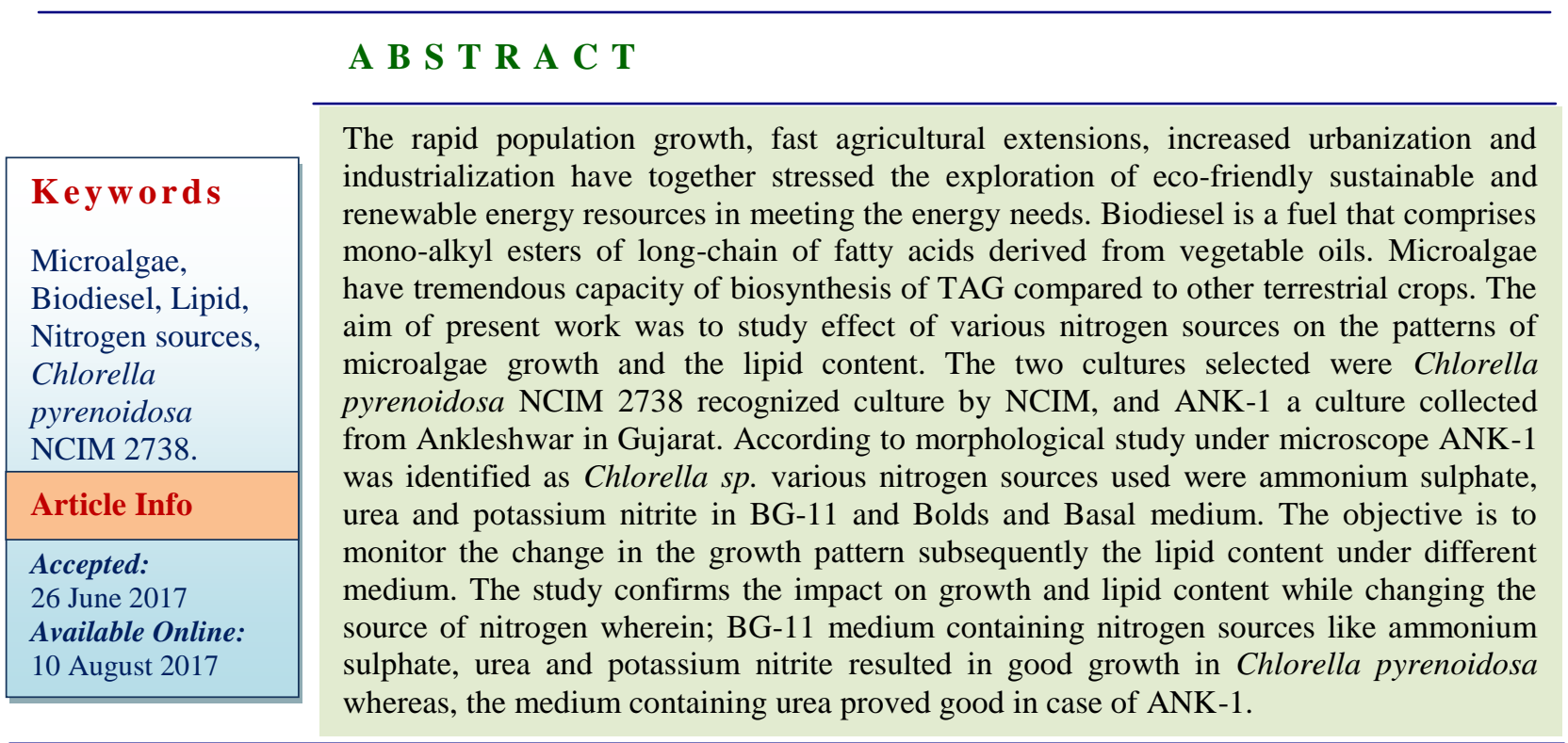

\section{Introduction}

The microalgae are unicellular photosynthetic organisms that use light energy and carbon dioxide, with higher photosynthetic efficiency than plants for the production of biomass (Benemann 1997, Miao, Wu, 2006). Microalgal lipids are regarded as the feedstock of future sustainable biodiesel production owing to much higher growth rates and photosynthetic efficiencies than conventional terrestrial plants (Chisti 2007; Li et al., 2008; Liu et al., 2008; Usui and Ikenouchi 1997). They may be destined to different applications, as biofuel production, purification of wastewater either autotrophic or mixotrophic conditions (Orús, et al., 1991,
Mũnoz, et al., 2006) extractions of high added value foods and pharmaceutical products, or as food for aquaculture (Spolaore, et al., 2006). The energy crisis has now become one of the biggest challenges of the 21st century. Oil and natural gas storage all over the globe have been estimated to be depleted in 40 and 64 years, respectively (Vasudevan and Briggs, 2008). Microalgae biomass contains approximately 50\% of carbon on a dry weight basis (Sánchez Mirón et al., 2003) and all the carbon present in cell usually from carbon dioxide. With the production of 100 tons of microalgae biomass, around 180 tons of $\mathrm{CO}_{2}$ can be 
disposed using natural or artificial light making the entire application as eco-friendly behavior. The lipids from microalgae could be used in different processes for energy exploitation, including the simple combustion in boiler or in a diesel engine. However, the best possible use of this oil is certainly its transformation to a biofuel, especially biodiesel (Chisti, 2007). Moreover, high added value compounds can be extracted from microalgae, such as fatty acids (linolenic, arachidonic, eicosapentaenoic, docosahexaenoic acids, etc.) (Cardozo, et al., 2007 and Valencia et al., 2007) pigments (carotenoids and ficobiliproteins), biochemically stable isotopes (Chisti 2007 and Borowitzka, et al., 1991) and vitamins such as biotin (Baker et al., 1981) vitamins C (S.A. Survase et al., 2006) and vitamin E (Running et al., 2002 and C. Bremus et al., 2006) besides some metabolites appear to have some pharmacological activities, among others the anticholesterolemic, antitumoral, immune modulatory, antibacterial and antimycotic ones. The biomass productivity, lipid cell content, and overall lipid productivity are some of the key parameters affecting the economic feasibility of algal oil for biodiesel production and associated buy products. Several studies have shown that the quantity and quality of lipids within the cell can vary as a result of changes in growth conditions temperature and light intensity or nutrient media characteristics concentration of nitrogen, phosphates and iron (IIIman et al., 2000 and Liu, 2008).

The lipids extracted from the two microalgae grown in Erlenmeyer flasks under conditions favoring lipid content in the cell have been analyzed quantitatively and qualitatively, by gravimetric and gas chromatographic methods, and different extraction methods have been investigated to maximize the yield in biodiesel. Nutrient limitation is an efficient environmental pressure to increase the lipid accumulation (Goldberg and Cohen, 2006 and Rodolfi et al., 2009). For instance, nitrogen limitation would cause three Changes; decreasing of the cellular content of thylakoid membrane, activation of acyl hydrolase and stimulation of the phospholipid hydrolysis. These changes may increase the intracellular content of fatty acid acyl-CoA. Meanwhile, nitrogen limitation could activate diacylglycerol acyltransferase, which converts acyl-CoA to triglyceride (TAG) (Takagi et $a l ., 2000)$. Therefore nitrogen limitation could both increase lipid and TAG content in microalgal cells. Many factors affect these properties, such as nutrient concentration (Aslan and Kapdan, 2006 and Goldberg and Cohen, 2006; Rodolfi et al., 2009), CO2 aeration (Chiu et al., 2009), light conditions (Solovchenko et al., 2008), and so on. The concentration of nitrogen and phosphorus present in water is considered to be the fundamental factor and has a direct influence on algal growth kinetics, which relates to nutrient removal and lipid accumulation closely. In present study was conducted to investigate the growth response and lipid content of a fresh water green algae NCIM 2738 Chlorella pyrenoidosa and ANK-1, by varying the nitrogen sources in different growth medium.

\section{Materials and Methods}

\section{Organism and growth conditions}

The culture of green alga Chlorella pyrenoidosa NCIM 2738 was provided by NCIM, India. The ANK-1 culture was collected from fresh water pond at Ankleshwar, Gujarat, India. These cultures were grown in $250 \mathrm{ml}$ Erlenmeyer flasks with $150 \mathrm{ml}$ medium. The medium used for cultivation was BBM and BG-11. The nitrogen source used was through the medium as urea, ammonium sulphate and potassium nitrate. The cultures were performed at 
thermally controlled algal laboratory at $25^{\circ} \mathrm{C}$ along with 16 hour fluorescent illumination (white tube light). The cultures were routinely hand shaken two to three times daily to avoid sticking. All the glassware's and media were sterilized prior to inoculation.

\section{Cell growth and biomass analysis}

Optical density measurement at $660 \mathrm{~nm}$ was used to monitor cell growth by UV/visible spectrophotometer with every three day time interval for 21 days. The total biomass weight was taken gravimetrically after 21 days. The biomass is harvested by centrifugation at $3000 \mathrm{rpm}$ for 10 minutes in $15 \mathrm{ml}$ Eppendorf tubes. The algal sample is kept in dried and pre-weighed crucibles. After drying sample crucibles were again weighed to know the total biomass of algae.

Total biomass $=\mathrm{C} 2-\mathrm{C} 1$

Where,

$\mathrm{C} 2$ is crucible with dried algae

$\mathrm{C} 1$ is empty pre-weighed crucible

\section{Lipid extraction}

\section{Microwave Digestion}

Digestion is the process in which the algal cell wall is denatured and the total lipid comes out. In microwave digestion method by using Microwave Digestive system the algal cell is digested. Pre-weighed dry powdered algal mass is taken in Teflon vessels along with $6 \mathrm{ml}$ of distilled water, in thoroughly washed Teflon vessels.

Teflon vessels are sealed with lids and loaded into the digestive system. These vessels are subjected to $175^{\circ} \mathrm{C}$ and 40 bar pressure. Time for 1 Cycle is taken as: 26minutes.
Lipid extraction from the Digested algal biomass was done using the method of Bligh and Dyer (1959) with some modifications. 2 $\mathrm{ml}$ methanol and $1 \mathrm{ml}$ chloroform was added to the dried algal biomass. The mixture was agitated on vortex for 2 minutes. The layers of the mixture were separated by centrifugation at $2000 \mathrm{rpm}$ for $10 \mathrm{~min}$. The supernatants collected and evaporation was carried out on heating mantle at $80^{\circ} \mathrm{C}$ till constant dryness was achieved.

Lipid content

$(\%$ dry cell weight $)=\mathrm{W} 2-\mathrm{W} 1 \times 100$

Where,

W1 is the weight of empty silica crucible and W2 is the weight of crucible + lipid

\section{Results and Discussion}

The time course biomass profile of Chlorella pyrenoidosa NCIM 2738 and ANK-1 grown autotrophically in BG-11 and BBM medium with different nitrogen sources (urea, ammonium sulphate and potassium nitrate) in the medium both in exponential and stationary phase. The original nitrogen source concentration in the medium was $1 \mathrm{~g} / \mathrm{L}$. Different nitrogen sources were used to investigate the effect on algal growth. Growth of algae increases from the $1^{\text {st }}$ day starting from log phase to stationary phase then decline phase till 24 days. Figure-1 explains that in nitrogen source as ammonium sulphate the ANK-1 shows long log phase while the Chlorella pyrenoidosa NCIM 2738 shows short log phase and long stationary phase. Figure- 2 shows that in urea as nitrogen source both Chlorella pyrenoidosa NCIM 2738 and $A N K-1$ show long log phase then stationary phase.Figure-3 highlights that in nitrogen source as potassium nitrate Chlorella pyrenoidosa NCIM 2738 and ANK-1 are showing the long log phase. 
The results interpret that the ammonium sulphate containing BG-11 the NCIM 2738 Chlorella pyrenoidosa shows higher optical density i.e. 1.70 while the ANK-1 shows optical density 0.60 after 21 days of incubation. The BG-11 that contains urea as nitrogen source brings out the information that the NCIM 2738 Chlorella pyrenoidosa mark with high growth bearing 0.65 optical density while ANK-1 growth is marked with the optical density as 0.46 after 21 days of incubation. Whereas, potassium nitrate containing BG-11 medium NCIM 2738 Chlorella pyrenoidosa shows fast growth rate with 0.39 optical density after 21 days of incubation while ANK-1 has 0.35 optical density with slower growth rate.

Figure. 4 explains that in nitrogen source as ammonium sulphate in BBM medium the Chlorella pyrenoidosa NCIM 2738 and ANK1 both are in log phase but after some interval of time the growth of Chlorella pyrenoidosa NCIM 2738 increases over the growth of
ANK-1. Figure. 5 shows that in nitrogen source urea Chlorella pyrenoidosa NCIM 2738 and ANK-1 both are in log phase. Figure. 6 interprets that in nitrogen source potassium nitrate the Chlorella pyrenoidosa NCIM 2738 show good growth initially in log phase and then enters stationary phase while ANK-1 is marked with very slow growth.

In case of BBM medium containing different nitrogen source the Chlorella pyrenoidosa NCIM 2738 and ANK-1 the Chlorella pyrenoidosa NCIM 2738 and ANK-1 have shown relatively same growth in ammonium sulphate contains BB medium with 0.21 and 0.27 optical density.

In case of urea both the sample cultures have shown relatively same growth but higher than ammonium sulphate i.e. O.D. is 0.79 and 0.78 respectively. Whereas in $\mathrm{BB}$ medium containing potassium nitrate as nitrogen source there is huge difference in growth i.e. optical density after ANK-1 has 0.37 .

Fig.1 Growth curve of Chlorella pyrenoidosa NCIM 2738 and ANK-1 grown on

BG-11 medium with ammonium sulphate as nitrogen source

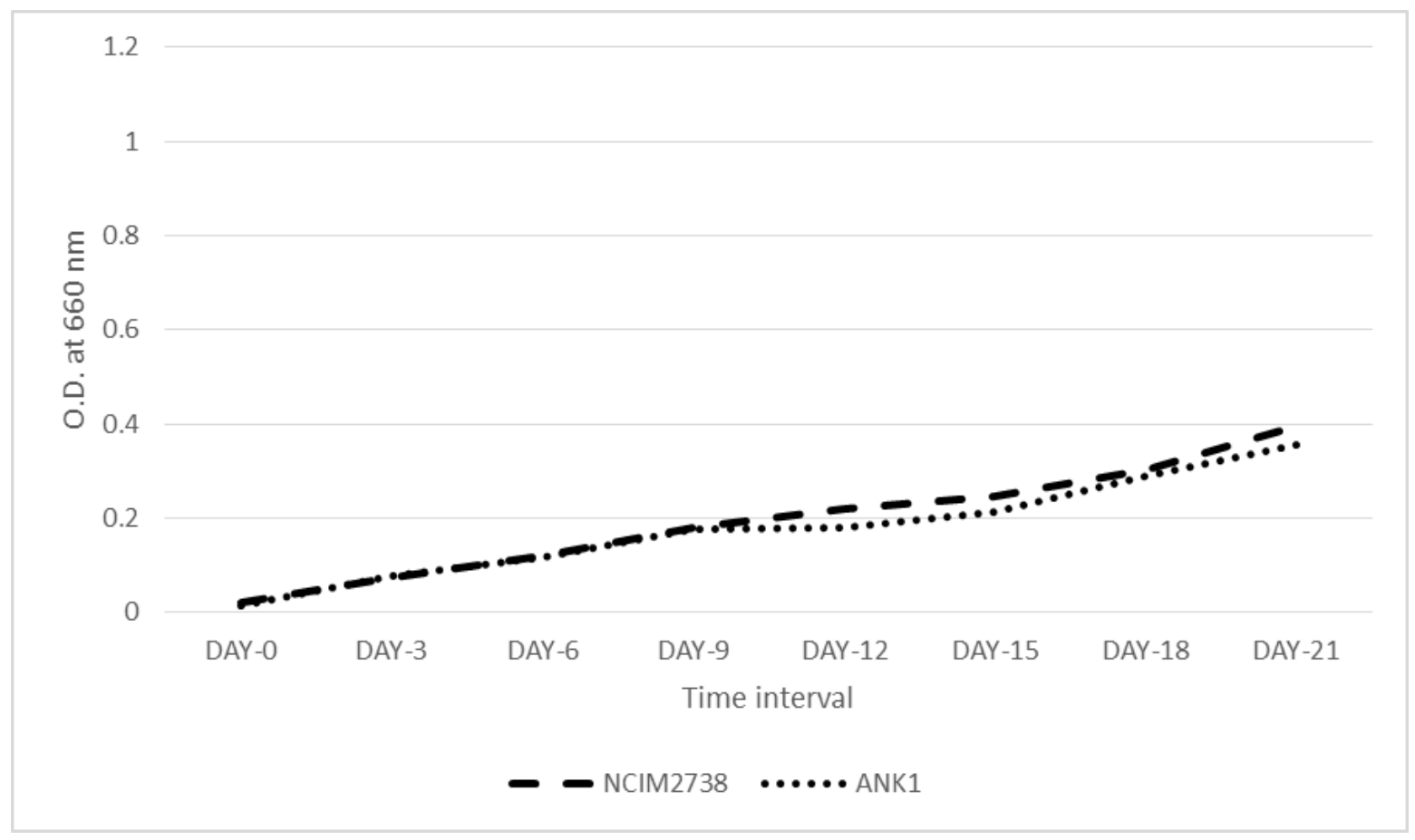


Fig.2 Growth curve of Chlorella pyrenoidosa NCIM 2738 and ANK-1 grown on BG-11 medium with urea as nitrogen source

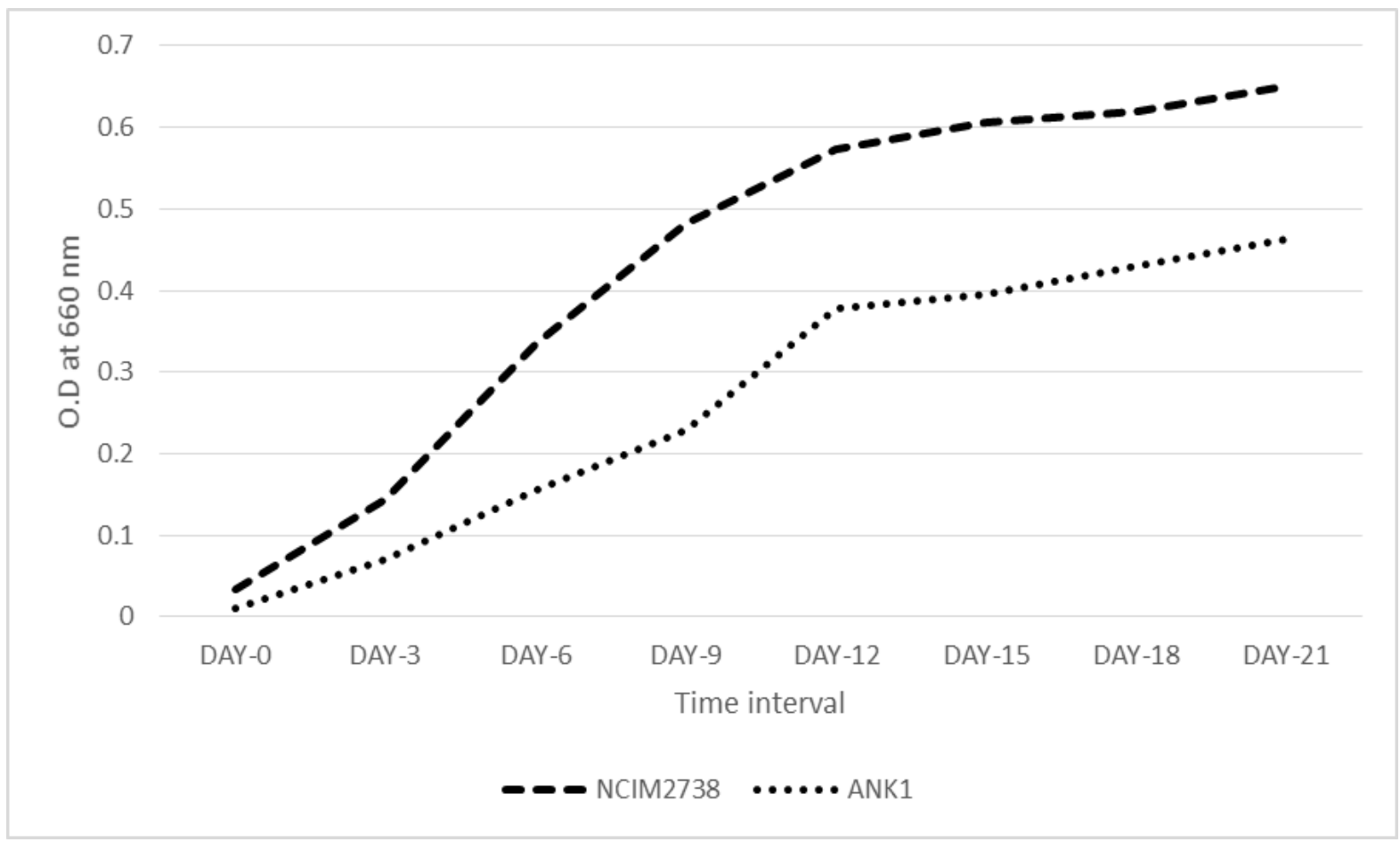

Fig.3 Growth curve of Chlorella pyrenoidosa NCIM 2738 and ANK-1 grown on BG-11 medium with potassium nitrate as nitrogen source

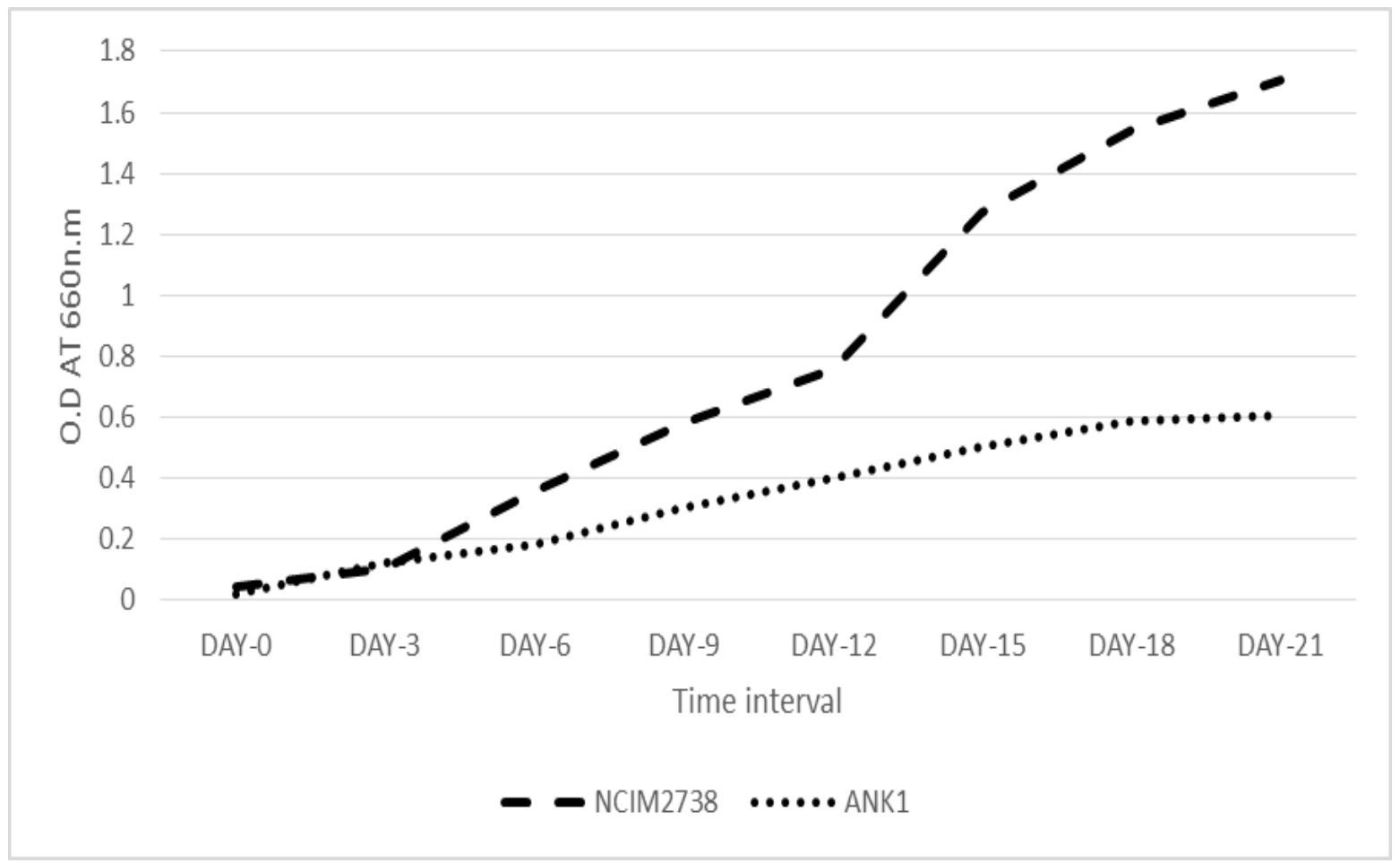


Fig.4 Growth curve of Chlorella pyrenoidosa NCIM 2738 and ANK-1 grown on BB medium with ammonium sulphate as nitrogen source

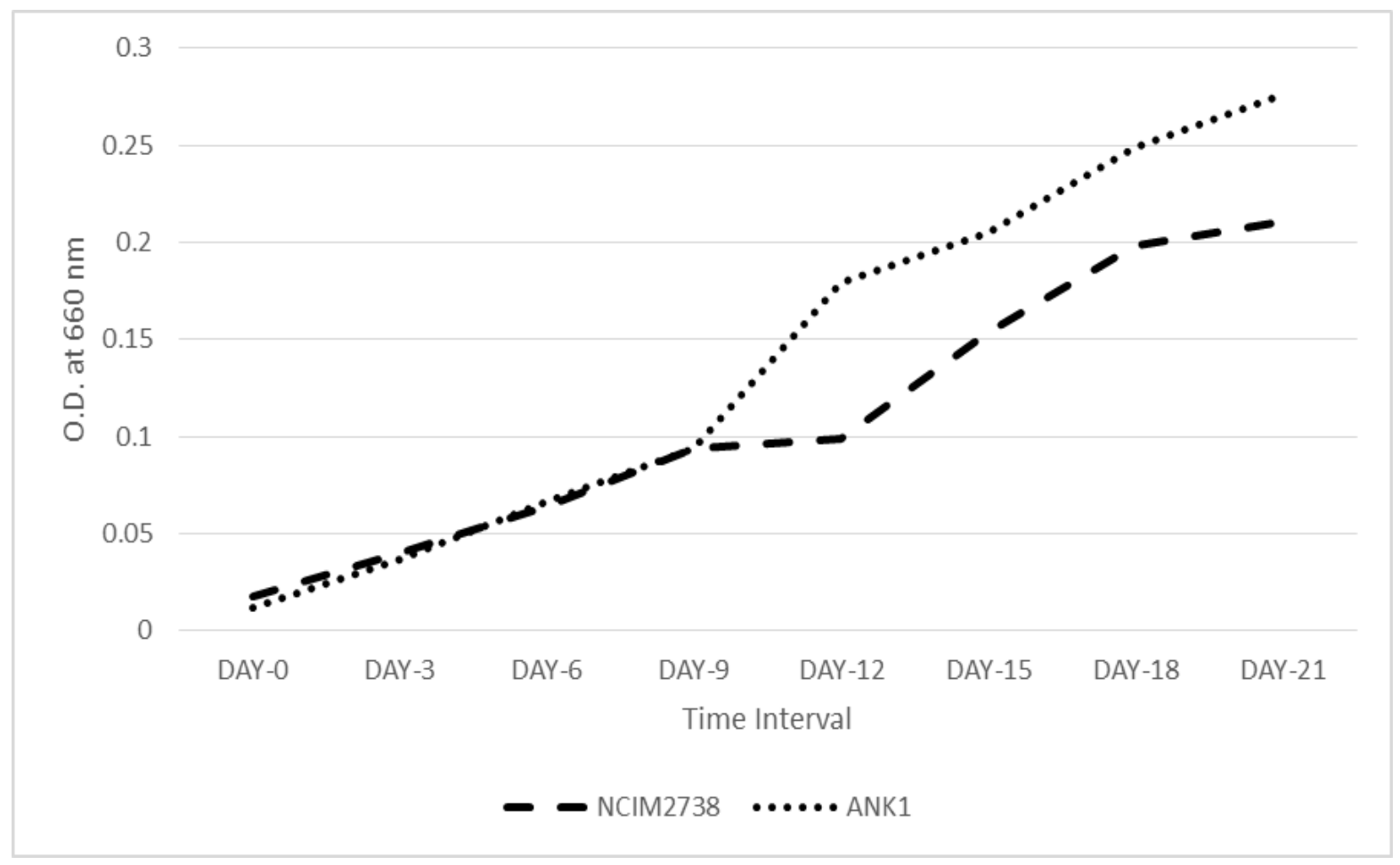

Fig.5 Growth curve of Chlorella pyrenoidosa NCIM 2738 and ANK-1 grown on BB medium with urea as nitrogen source

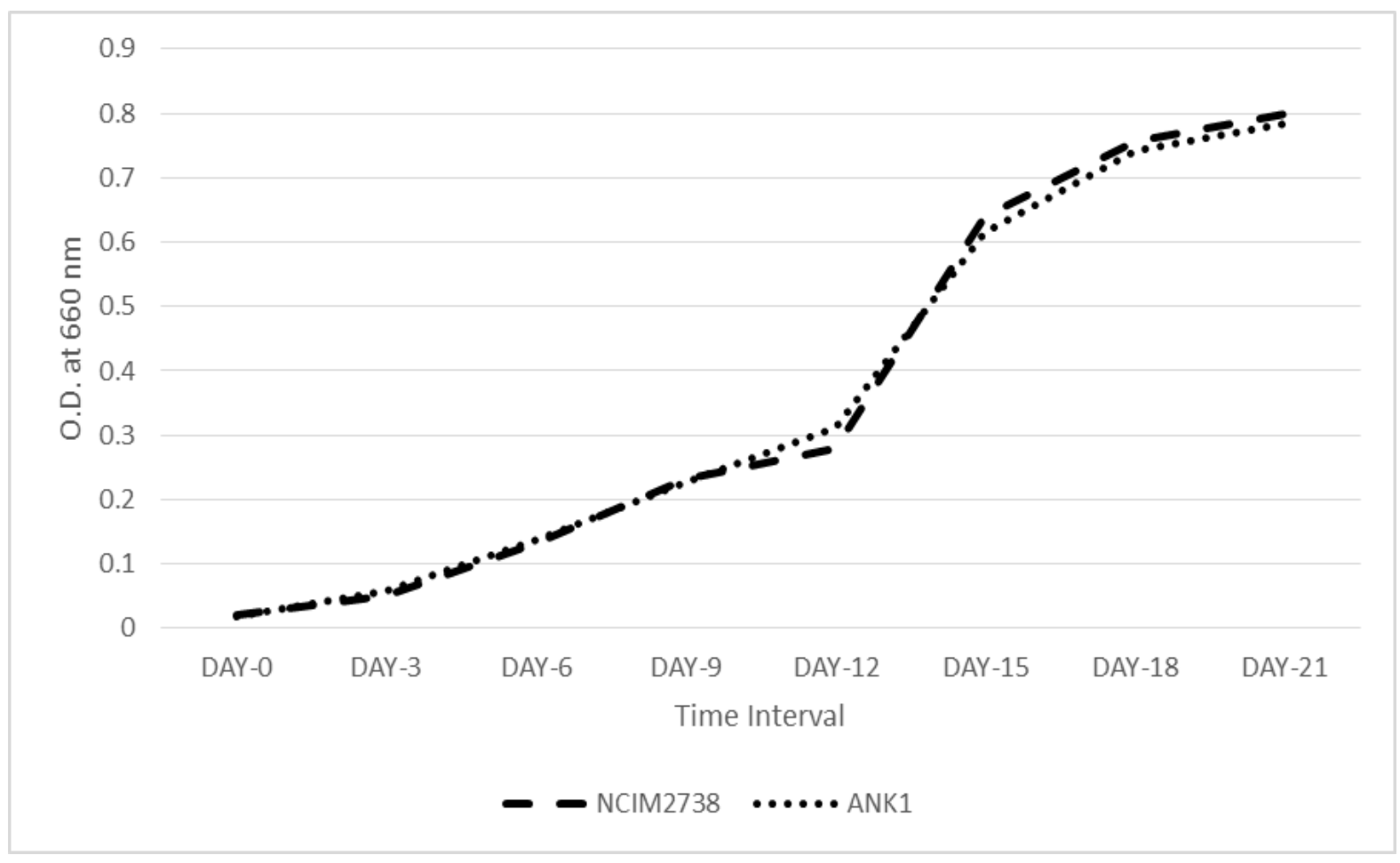


Fig.6 Growth curve of Chlorella pyrenoidosa NCIM 2738 and ANK-1 grown on $\mathrm{BB}$ medium with potassium nitrate as nitrogen source

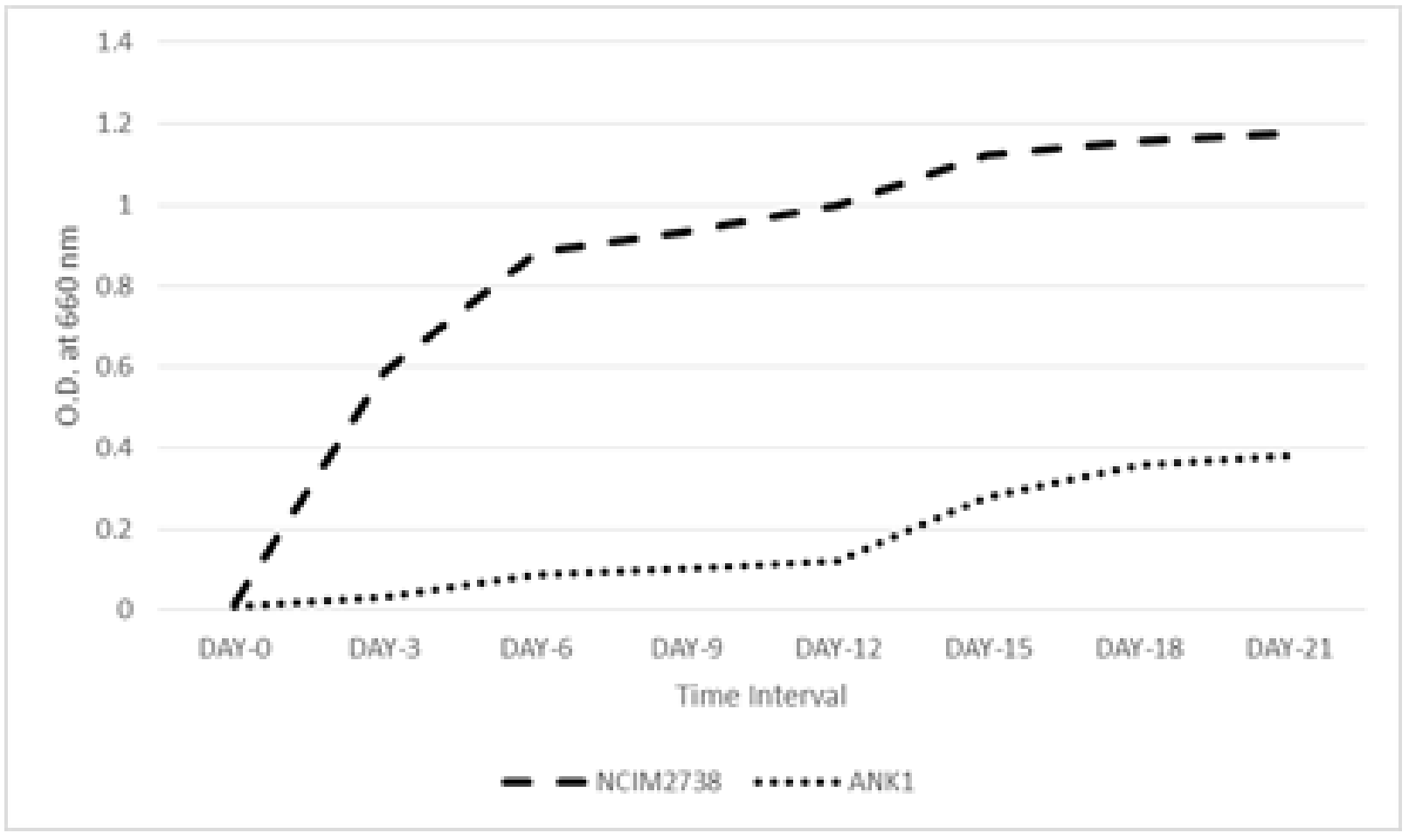

Fig.7 Comparison of lipid content of Chlorella Pyrenoidosa NCIM 2738 and ANK-1 grown in BG-11 medium with different nitrogen sources

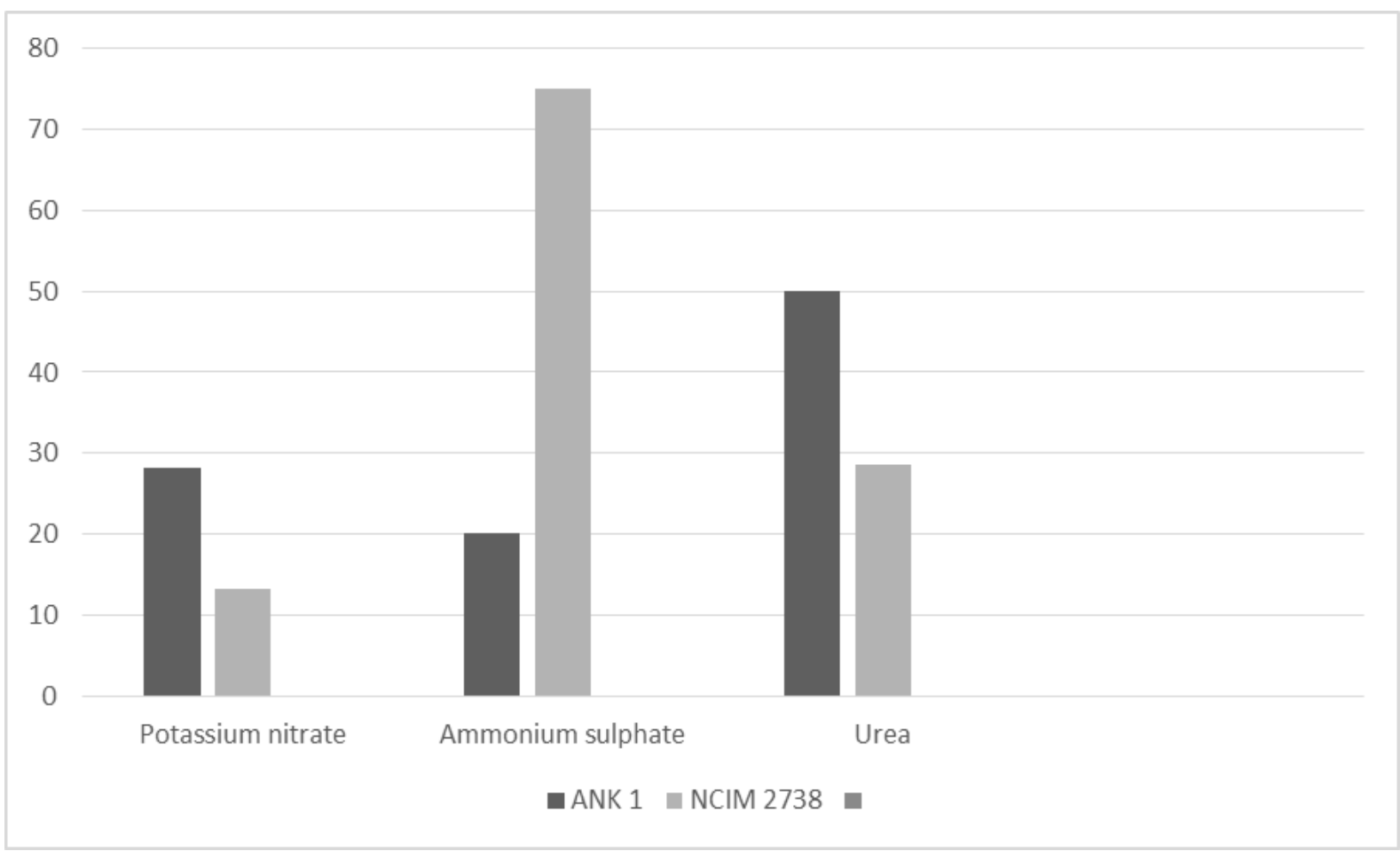


Fig.8 Comparison of lipid content of Chlorella Pyrenoidosa NCIM 2738 and ANK-1 grown in $\mathrm{BB}$ medium with different nitrogen sources

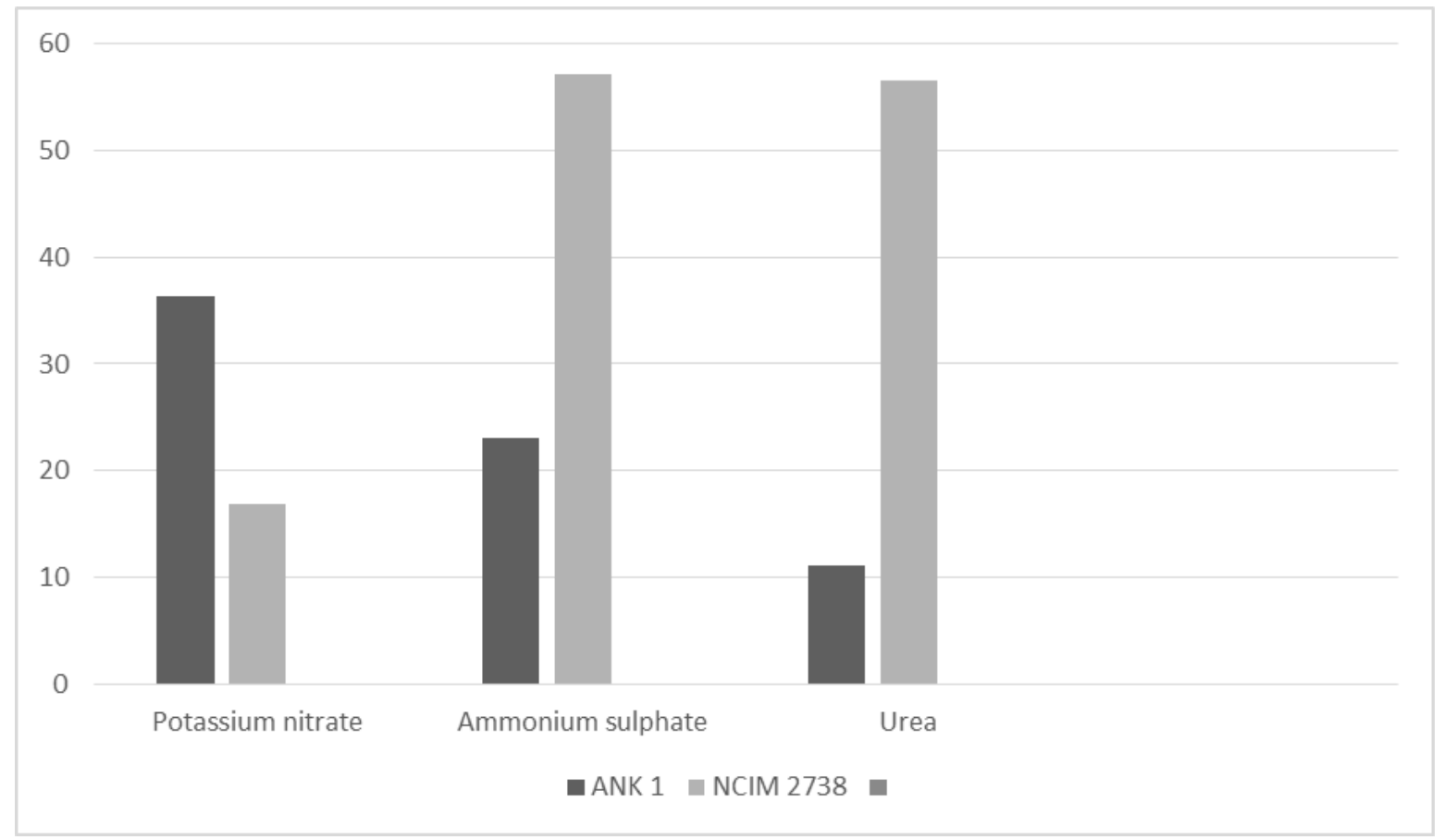

Table.1 Comparison of total biomass of in different nitrogen sources

\begin{tabular}{|c|c|c|}
\hline BG-11 & NCIM 2738 & ANK-1 \\
\hline Urea & $0.028 \mathrm{gm}$ & $0.01 \mathrm{gm}$ \\
\hline Potassium Nitrate & $0.076 \mathrm{gm}$ & $0.032 \mathrm{gm}$ \\
\hline Ammonium Sulphate & $0.008 \mathrm{gm}$ & $0.03 \mathrm{gm}$ \\
\hline
\end{tabular}

Table.2 Comparison of total biomass of in different nitrogen sources

\begin{tabular}{|c|c|c|}
\hline BBM & NCIM 2738 & ANK-1 \\
\hline Urea & $0.023 \mathrm{gm}$ & $0.108 \mathrm{gm}$ \\
\hline Potassium Nitrate & $0.071 \mathrm{gm}$ & $0.022 \mathrm{gm}$ \\
\hline Ammonium Sulphate & $0.007 \mathrm{gm}$ & $0.013 \mathrm{gm}$ \\
\hline
\end{tabular}

Figure 7 shows the lipid content of Chlorella pyrenoidosa NCIM 2738 in different nitrogen sources. wherein Chlorella pyrenoidosa NCIM 2738 show higher lipid in ammonium sulphate while ANK-1 show higher lipid in urea as nitrogen source in BG-11 medium. Figure. 8 highlights the BBM medium containing ammonium sulphate and urea Chlorella pyrenoidosa NCIM 2738 showing higher lipid content while the ANK-1 shows higher lipid in BBM medium containing potassium nitrate. In different organic and inorganic nitrogen source in BBM medium the lipid accumulation is higher in Chlorella pyrenoidosa NCIM 2738 ammonium sulphate i.e. $57.14 \%$. In BBM medium the lipid accumulation is higher in ANK-1 containing potassium nitrite as nitrogen source i.e. 36.36 $\%$. In different organic and inorganic nitrogen source in BG-11 medium the lipid accumulation is higher in Chlorella pyrenoidosa NCIM 2738 ammonium sulphate i.e. $75.00 \%$ while in ANK-1 higher lipid is found in urea i.e. $50.00 \%$. 
Biofuel can be produced economically and sustainably from micro-algae. Both biomass and lipid play an important role in biofuel production. Various studies are going on to enhance the algal growth and lipid content for biofuel production. Different nitrogen sources influence algal growth and lipid content differently i.e. Chlorella pyrenoidosa NCIM 2738 is showing good growth in urea and potassium nitrate as nitrogen source in BG-11 medium. ANK-1 is showing good growth in ammonium sulphate and urea as nitrogen source in BB medium, while showing little growth in potassium nitrate.

In conclusion, the present study suggests that most effective way to enhance microalgal growth and lipid is to provide proper nitrogen source. The most effective nitrogen source for Chlorella pyrenoidosa NCIM 2738 is urea and potassium nitrate while for ANK-1 the suitable nitrogen source is urea for growth. Chlorella pyrenoidosa NCIM 2738 show high lipid content in nitrogen source ammonium sulphate and urea whereas ANK-1 show high lipid in potassium nitrate and urea. However, if the observation time is extended the growth rate both in terms of biomass and lipid content will definitely be better than what has been observed during the course of this study.

\section{Acknowledgements}

The authors are thankful to the Director General at Gujarat Energy Research and Management Institute for moral support for the successful completion of this research work as part of student's internship program. The authors also acknowledge the support of the authorities of GUJCOST, CCD and GSBTM during the entire course this research work.

\section{References}

Baker, E., et al., 1981, Water-soluble vitamins in cells and spent culture supernatants of Poteriochromonas stipitata, Euglena gracilis, and Tetrahymena thermophila, Arch. Microbiol. 129, 310-313.

Benemann, J.R., 1997, CO2 mitigation with microalgae systems, Energy Convers. Manage. 38, 475-479.

Borowitzka, L.J., 1991, Development of Western Biotechnology's algal _carotene plant, Bioresour. Technol. 38, 251-252.

Bremus, C., U. Herrmann, et al., 2006, The use of microorganisms in 1-ascorbic acid production, J. Biotechnol. 124, 196205.

Cardozo, K.H.M., et al.,2007, Metabolites from algae with economic impact, Comp. Biochem. Physiol. C: Toxicol. Pharmacol. 146, 60-78.

Chisti, Y., 2007, Biodiesel from microalgae, Biotechnol. Adv. 25, 294-306.

Illman, A.M., et al., 2000, Increase in Chlorella strains calorific values when grown in low nitrogen medium, Enzyme Microb. Technol. 27, 631-635.

Illman, A.M., et al.,2000, Increase in Chlorella strains calorific values when grown in low nitrogen medium, Enzyme Microb. Technol. 27, 631-635.

Liu, Z.Y., et al., 2008, Effect of iron on growth and lipid accumulation in Chlorella vulgaris, Bioresour. Technol. 99, 4717-4722.

Liu, Z.Y., et al., 2008, Effect of iron on growth and lipid accumulation in Chlorella vulgaris, Bioresour. Technol. 99, 4717-4722.

Miao, X., and Q. Wu,2006 Biodiesel production from heterotrophic microalgal oil, Bioresour. Technol. 841-846.

Mũnoz, R., and B. Guieysse, 2006 Algalbacterial processes for the treatment of hazardous contaminants: a review,Water Res. 40, 2799-2815. 
Olaizola, M., 2003, Commercial development of microalgal biotechnology: from the test tube to the marketplace, Biomol. Eng. 20, 459-466.

Orús, M.I., et al.,1991, Suitability of Chlorella vulgaris UAM 101 for heterotrophic biomass production, Bioresour. Technol. 38,179-184.

Running, J.A. et al., 2002, Extracellular production of 1-ascorbic acid by Chlorella protothecoides, Prototheca species, and mutants of $P$. moriformis during aerobic culturing at low $\mathrm{pH}, \mathrm{J}$. Ind. Microbiol. Biotechnol. 29, 93-98.

Sánchez Mirón, et al.,2003, Shear stress tolerance and biochemical characterization of Phaeodactylum tricornutum in quasi steady-state continuous culture in outdoor photobioreactors, Biochem. Eng. J. 16,287-297.
Sawayama, S., 1996, CO2 fixation and oil production through microalga, Fuel. Energ.Abstr. 37, 217.

Spolaore, P., et al., 2006 Commercial applications of microalgae, 101, 87-96.

Survase, S.A., et al., 2006, Biotechnological production of vitamins, Food Technol. Biotechnol. 44, 381-396.

Valencia, D. Ansorena, And I. Astiasarán, 2007, Development of dry fermented sausages rich in docosahexaenoic acid with oil from the microalgae Schizochytrium sp.: influence on nutritional properties, sensorial quality and oxidation stability, Food Chem. 104, 1087-1096.

Yun, Y.S., et al., 1996, Carbon dioxide fixation by algal cultivation using wastewater nutrients, J. Chem. Technol. Biotechnol. 69 (1997) 451-455. F.B. Metting, Biodiversity and application of microalgae, J. Ind. Microbiol. Biotechnol. 17, 477-489.

\section{How to cite this article:}

Sweta M. Soni, Sunny S. Sankneniwar, M.A. Rasheed, P.L.S. Rao and Hasan, S.Z. 2017. Effect of Various Nitrogen Sources on Microalgal Growth and Lipid Content in Chlorella pyrenoidosa NCIM 2738 and ANK-1. Int.J.Curr.Microbiol.App.Sci. 6(8): 3099-3108. doi: https://doi.org/10.20546/ijcmas.2017.608.371 